\title{
Acknowledgements to Referees
}

Valerio De Angelis

\section{Abstract}

The quality of The Journal of Headache and Pain depends on the qualified and regular collaboration of renowned scientists, who devoted their time to constructively review the submitted articles.

We are indebted to the following experts who reviewed papers that completed the peer-reviewing process within 2014.

M.A. Al-Jumah

(Riyadh)

A.P. Andreou

(London)

A. Antal

(Göttingen)

F. Antonaci

(Pavia)

R. Arcioni

(Rome)

A. Ashkenazi

(Doylestown)

M. Ashina

(Copenhagen)

S. Ashina

(New York)

T. Bartsch

(Kiel)

E. Beghi

(Milano)

A.C. Belin

(Stockholm)

L. Bendtsen

(Copenhagen)

R. Benoliel

(Jerusalem)

L. Berliocchi

(Catanzaro)

M. Bjørn Russell

(Oslo)

A. Bozzao

(Rome)

Correspondence: valerio.deangelis@uniroma1.it

Assistant to the Editor, The Journal of Headache and Pain, Via di Grottarossa 1035, 00189 Rome, Italy

\section{F. Brighina \\ (Palermo) \\ O. Bruni \\ (Rome)}

J.M. Bugnicourt

(Amiens)

R. Cady

(Springfield)

L. Cao

(Biddeford)

S. Ceruti

(Milan)

S. Cevoli

(Bologna)

A. Charles

(Los Angeles)

T.C. Chaves

(Ribeirão Preto)

G. Coloprisco

(Rome)

G. Coppola

(Latina)

S. Cortese

(Verona)

C. Costa

(Perugia)

M. Davis

(Rochester)

M. de Tommaso

(Bari)

C. Di Lorenzo

(Latina)

V. Di Piero

(Rome)

\section{黑 Springer}

(ㄷ) 2015 De Angelis; licensee Springer. This is an Open Access article distributed under the terms of the Creative Commons Attribution License (http://creativecommons.org/licenses/by/4.0), which permits unrestricted use, distribution, and reproduction in any medium, provided the original work is properly credited. 
H.C. Diener

(Essen)

A. Donnet

(Paris)

J. Ellrich

(Aalborg)

M. Engstrøm

(Trondheim)

D. Fanelli

(Montreal)

C. Fernández-de-Las-Peñas

(Madrid)

M. Fioravanti

(Rome)

J.L. Fuh

(Taipei)

F. Galli

(Pavia)

A.R. Gantenbein

(Zurzach)

C. Gaul

(Essen)

P. Gazerani

(Aalborg)

J. Genizi

(Haifa)

G. Gentile

(Rome)

P. Geppetti

(Florence)

P.J. Goadsby

(San Francisco)

F. Granella

(Parma)

K. Hagen

(Trondheim)

J.K. Hesselink

(Utrecht)

J. Hoffmann

(Berlin)

D. Holle

(Essen)

T.P. Jürgens

(Hamburg)

M.A. Kabbouche

(Cincinnati)

D.G.A. Kasteleijn-Nolst Trenite

(Utrecht)

Z. Katsarava

(Unna)

S. Khuder

(Toledo)

M. Koutris

(Amsterdam)
T. Jürgens

(Hamburg)

G. Lambru

(London)

M. Lantéri-Minet

(Nice)

J.P. Lefaucheur

(Créteil)

V. Limmroth

(Cologne)

M. Linde

(Trondheim)

R. Lipton

(New York)

S. Maabjerg

(Glostrup)

F. Maggioni

(Padua)

E. Magiorkinis

(Athens)

D.L. Maier

(London)

N. Maleki

(Boston)

G.C. Manzoni

(Parma)

M. Marmura

(Phildadelphia)

M. Matharu

(London)

P. Matusz

(Lausanne)

A. May

(Hamburg)

J. McDougall

(Santa Rosa)

A. Milde-Busch

(Munich)

M. Monticone

(Pavia)

S. Nelson

(Boston)

A. Negro

(Rome)

A. Özge

(Yenişehir)

D.S. Paauw

(Seattle)

K. Paemeleire

(Ghent)

A. Paglialonga

(Milan)

S.L. Pan

(Taipei) 
J.A. Pareja

(Alcorcón)

P. Parisi

(Rome)

J. Pascual

(Santander)

J. Passchier

(Amsterdam)

D.B. Pfau

(Mannheim)

S. Prakash

(Baroda)

A. Raggi

(Milan)

I. Rainero

(Turin)

M. Robbins

(New York)

M.B. Russell

(Oslo)

S. Sacco

(L'Aquila)

F. Sakai

(Saitama)

P. Sandor

(Zurich)

P. Sarchielli

(Perugia)

A. Scher

(Bethesda)

E. Schmutzhard

(Innsbruck)

M. Schürks

(Essen))

G. Serafini

(Rome)

H. Shehata

(Cairo)

M. Sillanpää

(Turku)

A. Signore

(Roma)

M.M.S. Sindou

(Lyon)

N.N. Singh

(Columbia)

A. Siva

(Istanbul)

J. Smith

(Lexington)

A. Sonawalla

(Karachi)

A. Straube

(Munich)
O. Summ

(Münster)

T. Takeshima

(Osaka)

C. Tassorelli

(Pavia)

R. Teggi

(Milan)

P.C. Tfelt-Hansen

(Glostrup)

M. Thompson

(Toronto)

D. Toni

(Rome)

P. Torelli

(Parma)

A. Truini

(Rome)

B. Tsang

(London)

L. Valentinis

(Portogruaro)

M. Valeriani

(Rome)

A.M. Velly

(Montreal)

A. Verhagen

(Rotterdam)

G. Zanchin

(Padua)

D. Wagner

(Glasgow)

R. Walder

(Iowa City)

S.J. Wang

(Taipei)

$X$. Wang

(Bermont)

C. Wöber

(Vienna)

C. Wöber-Bingöl

(Vienna)

S.Y. Yu

(Beijing)

J.M. Zakrzewska

(London)

Received: 3 February 2015 Accepted: 3 February 2015 Published online: 27 February 2015 